\title{
Managing air pollution impacts to protect local air quality
}

\author{
C. Grant, R. Bloxam \& S. Grant \\ Ontario Ministry of the Environment, \\ Environmental Sciences and Standards Division, Canada
}

\begin{abstract}
Local industrial/commercial sources of air pollution in Ontario, Canada have been regulated for almost four decades using air emission estimating, atmospheric dispersion models and point of impingement (POI) standards. Historically, the provincial Ontario Ministry of the Environment (MOE) set standards that considered technical, economic and scientific issues. Compliance assessment used air emission inventories and atmospheric dispersion models originally developed in the 1960s. The challenges of this type of approach included:
\end{abstract}

- A cumbersome standard-setting process that produced few standards - often dictated by technical/economic considerations.

- Inaccuracies in air emission inventories.

- Dispersion models that tended to under-predict impacts.

In August 2005, Ontario announced a significant overhaul of the local air pollution regulation that included:

- Air standards that are now set to protect against health and environmental impacts.

- Phase-out of current dispersion models and replacement with the more accurate dispersion models from the United States Environmental Protection Agency (US EPA).

- Rigorous air emission estimating rules including the use of a combination of dispersion modelling and ambient monitoring as a more accurate emission estimating technique for a wide variety of sources (including fugitives).

- Technical/economic considerations that are now addressed through a publicly transparent alternative air standards process that promotes continuous improvement. Site specific alternative standards represent the lowest technically and/or economically feasible levels that a specific facility could achieve. Decisions often hinge on the technology benchmarking report, which is similar to the US EPA "top-down" analysis.

This paper outlines key challenges and policy decisions during the development of the regulation; experiences in introducing more stringent scientific-based standards, including standards for lead and vinyl chloride, which are among some of the most stringently regulated standards in the world; and lessons-learned in the use of the combined monitoring and modelling emission estimating tool in the new alternative standards process.

Keywords: local air quality, air toxics, dispersion modelling, monitoring, technical and economic barriers, air standards, fugitive emissions. 


\section{Historical background}

Ontario, Canada is home to a wide range of heavy industries including sectors such as iron and steel, petroleum refining, chemical production, pulp and paper, mining and primary metal smelting, as well as a large automotive sector. The Ontario Ministry of the Environment (MOE) has regulated local air pollution since the late 1960s. It was one of the first provinces in Canada to regulate air pollution. The province sets contaminant-specific Point of Impingement (POI) air standards to manage air pollution from industrial and commercial sources. Mathematical air dispersion models have been the primary tools used to assess compliance with air quality standards along with occasional ambient monitoring in specific communities. The initial regulation set out simple Gaussian air dispersion models, which were considered state-of-the-art at that time. Outputs from the models are used to compare POI concentrations to the air standards: ambient monitoring could also be used. Ontario's reliance on POI air standards is somewhat unique where many other jurisdictions emphasize air emissions or best available control technology standards. For example, since the Clean Air Act Amendments of 1990, the United States has used a combination of Maximum Achievable Control Technology (MACT) standards and state-specific requirements to regulate air toxics (US EPA [1]). As other jurisdictions moved to regulating technology or emission standards - as opposed to concentration based POI standards - Ontario began a review of its regulatory framework and considered similar approaches. However, proposed changes created uncertainty for industry and raised questions about technical barriers to compliance and costs. In 2001, Ontario began a new consultation process. The key drivers for change were based on criticisms that the current air standards were over 20 years old and were not protective of health and environmental impacts and that current air dispersion models were over 30 years old and could be underestimating concentrations. For many years, Ontario had challenges in setting and updating air quality standards and updating its air dispersion models since:

- a change in the air standard for one substance could affect a variety of sectors in different ways;

- more stringent air standards can create technical and economic implementation issues for industry;

- changes to the air dispersion models, used to assess POI concentrations, could lead to potential compliance issues;

- uncertainty in assessing compliance could result in permitting delays which could lead to uncertainty in making business decisions;

- the past practice of setting an air standard that considered economics, technology and science was complex; lacked public transparency; resulted in less protective standards; and took significant time and resources for each standard.

\subsection{A new legal framework to protect local air quality}

In August 2005, Ontario announced a significant overhaul of the local air pollution regulation - Ontario Regulation 419/05: Air Pollution - Local Air 
Quality (hereafter referred to as the "Regulation"): further amendments were announced in 2007 (MOE [6]). The new regulatory framework combined science-based air quality standards with the use of technology standards (similar to the US EPA [1]) as an alternative if compliance with air standards could not be achieved in the short term. This Regulation is the primary tool used to protect local air quality by enforcing air quality standards. One of the key policy shifts that occurred as a result of the Regulation was a move towards provincial air quality POI standards that were set to protect against health and environmental effects - as opposed to setting air standards that considered technical and economic concerns (MOE [3]). Under the new regime, the past practice of relaxing standards based on the concerns of one industry or a specific sector would not continue. This Regulation now includes:

- 59 new or updated air standards set to protect against health and environmental impacts - new or more stringent standards are phased-in.

- Phase-out of current atmospheric air dispersion models and replacement with the more accurate dispersion models from the United States Environmental Protection Agency (US EPA).

- Rigorous emission estimating rules that include a combination of dispersion modelling and ambient monitoring as a more accurate method to assess emission rates for a wide variety of sources.

- A new risk-based decision making process that considers technical and economic barriers through a publicly transparent alternative air standards process, which promotes continuous improvement. Site specific alternative standards represent the lowest technically (and/or economically) feasible levels that a specific facility could achieve. Decisions often hinge on a technology benchmarking report, which is similar to the US EPA "top-down" analysis (US EPA [4]).

Implementation issues that result from the updating of air quality standards and air dispersion models can be addressed by allowing a facility to request an alteration of the standard to address site specific technical and/or economic concerns through a publicly transparent process. Ontario's regulatory framework has evolved into an effective hybrid of the POI approach using effects-based standards and a process that allows for site specific technology standards if needed to address compliance issues and promote continuous improvement.

\section{Emission estimating methods}

One of the items that the Regulation addressed was uncertainties in emission estimation methods that are inherent in any air dispersion modelling approach. The regulatory framework requires a facility to determine, for any given source, an emission rate that represents the highest emission that a facility is capable of based on a given operating condition. In order to expedite compliance assessments, a facility may initially use a "conservative" emission estimate to determine the maximum off-property POI concentration. If this maximum POI concentration complies with the air standard, no further assessment is required. If a facility exceeds the standards at those levels, then they are required to 
"refine" their emissions to be as accurate as possible. Refinement of emissions must be done in one of two ways:

- Estimating emissions based on a combined modelling and monitoring analysis; or

- Source testing across a range of operating conditions may also be considered but only if it is specifically approved as a more accurate or effective approach than a combined modelling and monitoring analysis.

Alternatively, the regulation allows a facility to move directly towards abatement improvements instead of complex studies to determine emission rates.

\subsection{The combined modelling and monitoring method}

Typically, source testing is conducted at a maximum operating condition that gives rises to a conservative emission rate with the premise that other operating conditions would result in lower emission rates (and hence a lower POI concentration). However, source testing is often conducted under optimal conditions and these emission rates may not be representative. In addition, source testing for fugitive emissions, which are often underestimated, is not possible. A more accurate assessment of emissions uses a combination of air dispersion modelling along with ambient air monitoring. Monitors are strategically located to determine emissions rates from key dominant sources of that contaminant for each monitoring period. It is important to locate monitors close enough to the source to capture all emissions, in particular fugitive emissions. However, if there is any uncertainty in source release parameters care should be taken not to locate monitors too close to the source. Verification of source release parameters for fugitive sources requires careful location of monitors. Siting considerations for the monitors is important and will include nearby proximity to the most significant sources of air emissions; site-specific geometry that may affect the dispersion of the contaminants from the source; obstructions to the monitor that may affect collection of the ambient air sample; and the predominant wind direction. This type of ambient monitoring program is often focused on collecting samples of contaminants immediately downwind of the key sources of contaminant and generally ends after collection of approximately thirty samples that are significantly above "background" levels of the contaminant. The approach is particularly well suited for estimating fugitive air emissions from sources of both particulate matter and volatile organic compounds but can also be used for other sources. The results of the ambient monitoring are used in combination with an iterative approach to revise the air emission rates for the dominant sources until the dispersion modelling results match the ambient monitoring results. In summary, a combined monitoring and modelling analysis recommends the following steps (MOE [5]):

i) Approval by the Ontario MOE of a combined monitoring and modelling plan that includes pre-agreement of the appropriate source of meteorological data for the dispersion modelling; appropriate siting of monitors and recording of relevant facility operating parameters during the monitoring periods (e.g., generally, a 24-hour monitoring period). 
ii) Installation of monitors and gathering of samples on a regular basis or when meteorological forecasting indicates that the monitors will be downwind of the main sources of a contaminant.

iii) A preliminary analysis of the monitoring data, the predominant wind directions and wind speeds for the monitoring period and the facility operating conditions to select the most significant measurements for further analysis and refinement of air emission estimates.

iv) Application of the atmospheric dispersion models for each of the monitoring locations (plus four locations in the immediate vicinity of each monitor to average any spatial anomalies between the monitor and modelling locations) using the meteorological data inputs for the specific monitoring period.

v) Results that are paired in time can be plotted on logarithmic paper of modelling versus monitoring data. Air emission estimates, for the most dominant and uncertain sources, are modified by iteration that can be focused on the most significant emission sources where the estimates are uncertain.

vi) Monitoring and modelling data is then re-organized, unpaired in time, from highest to lowest for each of the monitoring and modelling data.

vii) The revised emission estimates provides an indication of the variation in air emissions, from the most significant contributors to the measured concentrations, during the monitoring study. For log-normal-like distributions of data, the use of the mean plus one standard deviation for the most significant sources identified from the study in combination with average emissions from all other sources is recommended.

Field costs for a combined monitoring and modelling analysis can be comparable or slightly less than the costs for a comprehensive source testing program. Computer run times for the dispersion modelling are relatively short but the required analysis between iterations can be resource intensive. To test this methodology, MOE conducted a study of lead emissions from a large brass and bronze foundry in southern Ontario. Figure 1 provides a plot of the initial unpaired monitoring versus modelling data for measurement of lead concentrations in the immediate vicinity the foundry. The modelled data was derived using an existing air emission inventory for the facility based primarily on source testing. This initial plot shows a trend towards the right-hand side of the plot, which has been interpreted by MOE as a possible under-estimating of POI concentrations and air emissions at the higher measurements (MOE [6]). Other combined analysis projects are underway for chemical plants, integrated iron and steel mills, smelting operations, and petroleum refineries.

\section{Implementing air quality standards}

Many jurisdictions recognize the need to protect human health and the environment while acknowledging the importance of goods and services provided by industry. The risk-based alternative standards process provides a mechanism to balance these interests in a publicly transparent manner. Ontario's 


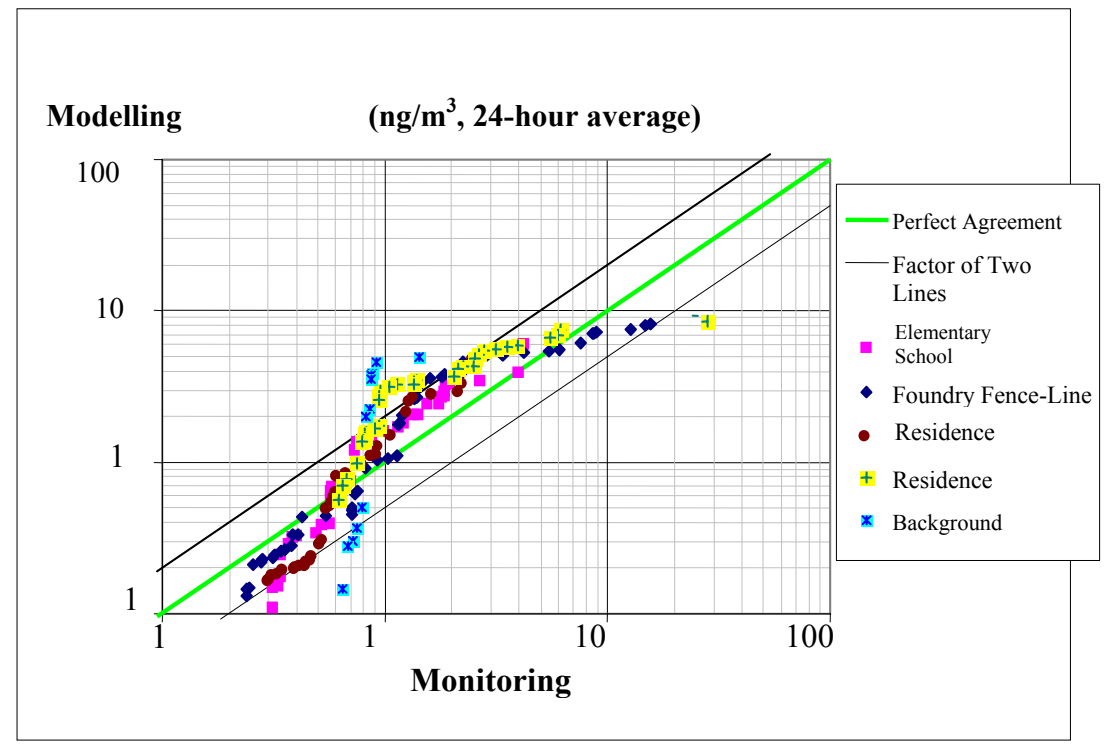

Figure 1: AERMOD model vs. monitoring for lead emissions.

risk-based framework for air quality standards is presented in Figure 2. The inverted triangle represents a measure of "risks". Risks generally increase at higher POI concentrations or exposures - the frequencies of those exposures may also be a consideration (MOE [3]). Provincial air quality standards that are set to protect against health and environmental effects are represented by the lower level line, below which risks are considered generally acceptable (Zone 1: "Broadly Acceptable Region"). The MOE's objective for air standards for carcinogenic effects is to set the standard that corresponds to an incremental lifetime risk of 1 in a million $\left(10^{-6}\right)$ (i.e. the risk of one person in a population of a million who may develop some form of cancer). Standards for noncarcinogenic effects are based on a Reference Concentration (RfC) that is derived from a threshold toxicological endpoint (the most sensitive) and the use of uncertainty factors to account for gaps in the data. Generally, the objective for non-carcinogenic risk is to set the standards based on a Hazard Quotient (HQ) of one, which is the ratio of the concentration of a contaminant to the corresponding air standard or RfC. The framework also defines an "Upper Risk Threshold" (shown as the upper level line - Zone 3). Concentrations in this region require timely action to assess and if necessary, to reduce contaminant concentrations as soon as possible. URTs for carcinogens are generally set at a 1 in 10,000 risk level $\left(10^{-4}\right.$ or 100 times the standard); URTs for non-carcinogens are generally set at a HQ of 10 (or 10 times the standard). POI concentrations between the upper and lower levels are in the "Region of Concern" (Zone 2). Facilities operating in this Region are required to take all reasonable steps to get into compliance with the effects-based standard by the phase-in date. If compliance 
with a standard is not possible by the phase-in date, these facilities may be eligible to request a site specific alternative standard - which is a risk-based approach (CSA [7], McColl et al [8]).

\section{Overview of the Alternate Standards Process}

\section{As low as reasonably achievable (ALARA) principle}

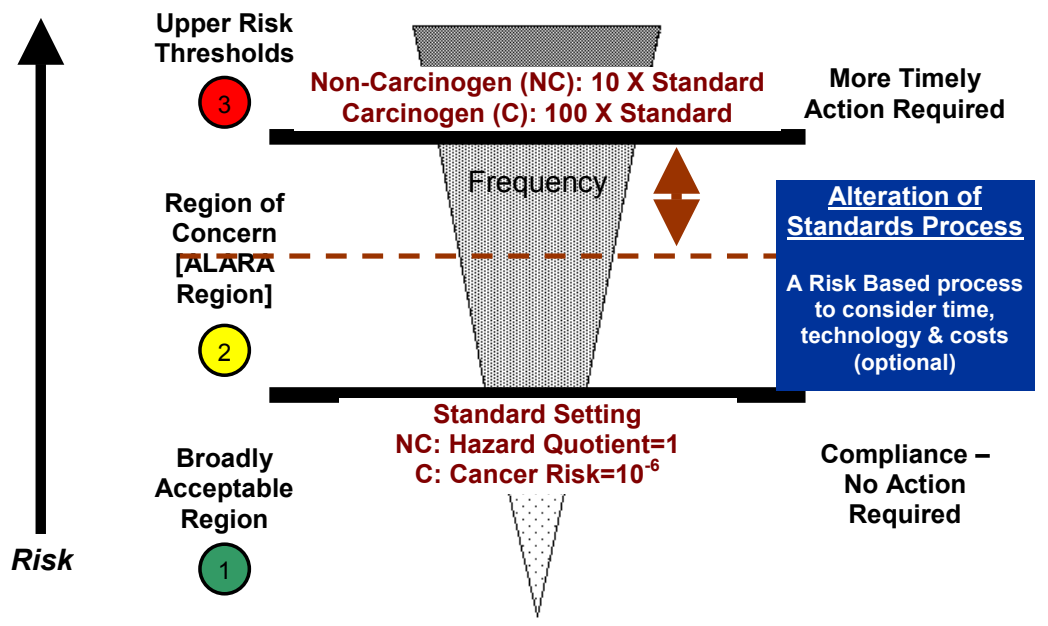

Figure 2: $\quad$ Risk framework for air quality standards.

\subsection{The alternative standards process}

Introduction of effects-based air standards, and more advanced air dispersion models, meant that not all facilities would be able to achieve compliance immediately due to technical limitations, economic realities or the need for more time to assess, plan, and if necessary, finance and install new equipment or processes to come into compliance. In these cases, the goal is to strive for reducing risk to "As Low As Reasonably Achievable" (ALARA). These issues could be reviewed as part of the site specific alternative standards process set out in the Regulation. An alternative standard will not be considered if the URT is exceeded at receptors such as daycares, schools, dwellings, hospitals or homes for the elderly. The frequency of exceedances is also a consideration (MOE [3]).

For most facilities, a phase-in period provides time to assess, plan, budget and implement technical solutions to ensure compliance with the air standards. Phase-in periods for new or more stringent standards are normally 3 to 5 years, but can vary based on the contaminant. If a facility can identify feasible technical solutions that can be implemented within the phase-in period to achieve compliance, then it should proceed to do so. For other facilities, compliance with air standard(s) might not be achievable within the phase-in period. If the 
technical solutions are not readily available to allow a facility to achieve compliance before the end of the phase-in period, these facilities may consider requesting Director's Approval for a site specific alternative standard. An alternative standard would establish an interim site specific standard with the goal of continuous improvement toward achieving the effects-based standard over time. When making a request for an alternative air standard, industry must include in their request:

- An Emission Summary and Dispersion Modelling (ESDM) report using emission rates determined from a modelling and monitoring analysis;

- A technology benchmarking report, comparing the facility to others within the sector to ensure they are doing the best they can;

- Summary comments from a public meeting held in the community; and,

- An action plan to minimize POI concentrations.

If approval is granted, the decision would be periodically reviewed to ensure that the technical (or economic (optional)) issues considered at the time are still relevant for that particular facility. The Director may approve a site specific alternative standard for a period of up to 5 years (up to 10 years in extenuating circumstances). A facility is eligible to re-apply but the Director must consider the number of times a request has been made for an alternative standard and the subject of the request. This will be considered on a case-by-case basis.

\subsection{Technical and economic considerations}

The alternative standards process allows for consideration of technical and economic feasibility in separate analyses. Technical feasibility is a mandatory component of the process and is documented in a "Technology Benchmarking" report. Economic feasibility is optional. Any information submitted as part of the request must also be shared with the local community if requested.

\subsubsection{Technology benchmarking reports}

A Technology Benchmarking Report is a key document submitted to support a request for an alternative standard. A list of process and site-specific air pollution control strategies are assessed in a prescriptive approach that is based upon the US EPA New Source Review “top-down” analysis (US EPA [4]). Various pollution control options are ranked from most to least effective at controlling emissions and POI concentrations. In practice, the technology benchmarking process may be simplified by focusing on the priority contributors to POI concentrations identified through the combined monitoring and modelling analysis. MOE is the midst of reviewing alternative standard requests for two polyvinyl chloride (PVC) chemical plants in southern Ontario. MOE's review of the technology benchmarking reports included the following components:

- A review of requirements for similar facilities in other jurisdictions (e.g., the U.S, Europe and Australia);

- A review of the performance of the Ontario facilities (grams of total/fugitive pollutant emitted per tonne of product produced) relative to information published on similar facilities in other jurisdictions; and 
- Third party technical experts to assess feasible technical methods for the key sources identified through the combined monitoring and modelling analysis.

Figure 3 provides a summary of the outcome of the Ontario MOE review of the technology benchmarking report and request for alternative standard for one facility. The graphic includes the relative performance (in grams per tonne of product produced) of the facility from 1994-1999 and in 2007.

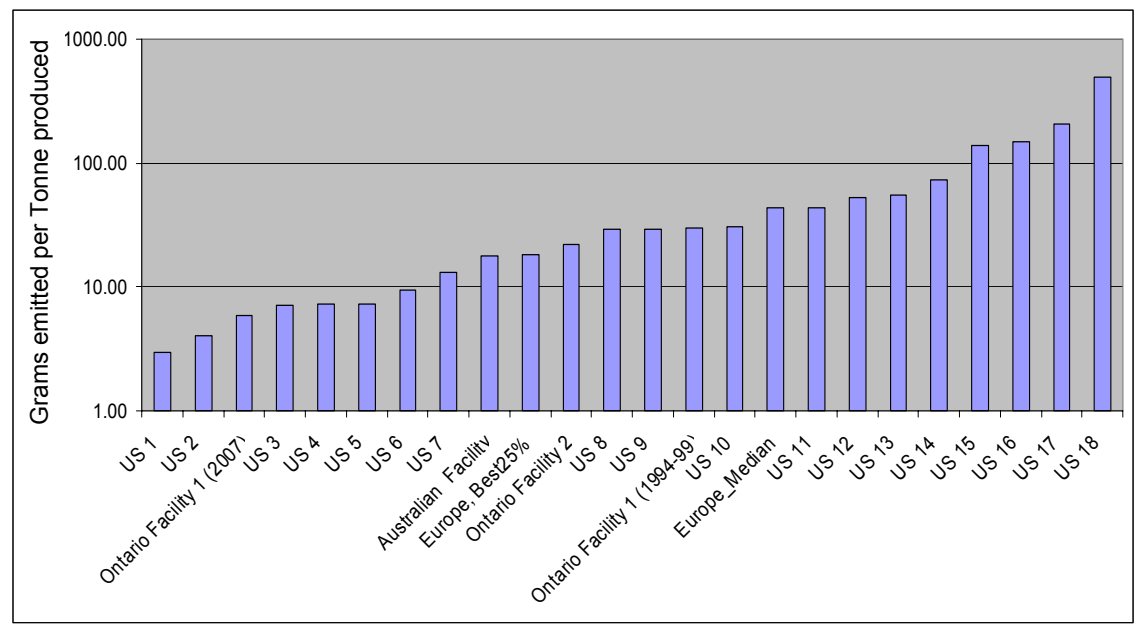

Figure 3: Benchmarking of Ontario facility.

\subsubsection{Financial hardship and cost effectiveness}

The alternative standards process also allows for the consideration of economic feasibility. MOE guidance includes economic ratios to assist in determining financial hardship. MOE is also considering development of cost effectiveness indicators which contemplates using traditional parameters, such as a cost per unit emission rate, and traditional pollution control costing techniques, such as those recommended by the US EPA (US EPA [9]), that are modified to account for relative toxicity of the contaminant; type of emission source (e.g., point, volume or area fugitive sources) and the magnitude and frequency of exceedances.

\subsubsection{Ongoing challenges to implementation}

The Regulation is primarily designed to address air toxics from larger industrial facilities. Smaller to medium sized facilities may find the regulatory approach burdensome. Alternatively, sector specific approaches are being considered for sectors that have common air pollution issues. Other on-going challenges include the need to address concerns about the cumulative effect of multiple facilities and contaminants within an air shed. 


\section{References}

[1] US EPA Clean Air Act Amendments, 1990 http://www.epa.gov

[2] Ontario Regulation 419/05:Air Pollution-Local Air Quality www.ene.gov. on.ca

[3] MOE. 2005. Guideline for the Implementation of Air Standards in Ontario, Toronto, Ontario. Queen's Printer for Ontario, PIBs \#5166e http://www.ene.gov.on.ca/envision/gp/5166e.pdf

[4] New Source Review Workshop Manual, Prevention of Significant Deterioration and Nonattainment Area Permitting, Draft, October 1990" Chapter B, Best Available Control Technology (top down analysis) www.epa.gov/ttn/nsr/gen/wkshpman.pdf

[5] MOE. 2005. Procedure for Preparing an Emission Summary and Dispersion Modelling (ESDM) Report, Toronto, Ontario. Queen's Printer for Ontario, PIBs \#3614e02 http://www.ene.gov.on.ca/envision/gp/3614e02.pdf

[6] MOE. 2007. Draft Report for an Air Emissions Study and Development of "An Accurate As Possible" ESDM Report for a Brass and Bronze Foundry with contributions from Senes Consultants and Ortech Environmental

[7] CSA. 1997. Risk Management: Guideline for Decision Makers. Canadian Standards Association, CAN/CSA-Q850-97 (R2002)

[8] McColl S, Hicks J, Craig L, and Shortreed J. 2000. Environmental Health Risk Management - A Primer for Canadians. Network for Environmental Risk Assessment and Management (NERAM). Waterloo, ON N2L 3G1

[9] US EPA Control Cost Manual, Office of Air Quality Planning and Standards, Research Triangle Park, NC 27711, EPA 453/b-96-001 